\title{
Day surgery: development of a national comparative audit service
}

\author{
Nick Black, Mark Petticrew, Duncan Hunter, Colin Sanderson
}

\begin{abstract}
Objectives-To develop software for hospitals to analyse their own survey data on patients' experiences of day surgery and to create and test the feasibility of a national comparative audit service.
\end{abstract}

Design-Software development and testing; database analysis.

Setting-Eleven general hospitals in England.

Patients-1741 day surgery patients undergoing procedures during 1991-2.

Main measures-Postoperative symptoms, complications, health and functional status, general satisfaction, and satisfaction with specific aspects of care. Results-Software for data entry and analysis by hospitals was successfully used at the pilot sites. The overall response rate for the 11 hospitals using the questionnaire was $60 \%$, ranging from $33 \%$ to $90 \%$ depending on the way the survey was managed. Data from all 11 hospitals were included in the national comparative audit database. Hospitals showed little variation in measures of patients' overall satisfaction (around $85 \%$ ), but significant differences were apparent for specific aspects such as receiving adequate written information before admission (range $50 \%-89 \%$ ), provision of adequate parking facilities $(14 \%-92 \%)$ and experiencing a significant amount of postoperative pain $(8 \%-42 \%)$. The proportion of day case patients undergoing procedures that could have been performed in outpatient departments varied from 0 to $27 \%$ between hospitals. Further comparisons of outcome, in particular measures of effectiveness, must await the development of validated case mix adjustment methods. Conclusion-Establishing a comparative audit database is feasible but several methodological problems remain to be resolved.

(Quality in Health Care 1993;2:162-166)

\section{Introduction}

The development of a questionnaire suitable for assessing patients' experiences of day surgery has been described in the preceding paper (p 157). ${ }^{1}$ Its use provides clinicians and managers with a description of their patients' views at a single time point. By identifying areas of poor outcome or satisfaction, it can enable appropriate action to be taken to improve the quality of day surgical care. The questionnaire can then be used to reassess the situation to see whether the expected improvements have occurred, thus completing the audit cycle. However, this scenario supposes that the clinicians and managers have been able to set appropriate standards. For example, they need to know what proportions of patients might reasonably be expected to complain of postoperative pain or be dissatisfied with the amount of written information they received before admission. For this, hospitals need to be able to compare their own findings with those obtained in other hospitals.

This need can be met by the widespread adoption of the same questionnaire by many hospitals and the construction of a national comparative database by aggregating the results centrally. Comparative audit in surgery is not a new concept, ${ }^{2}$ though there are few established examples in the United Kingdom..$^{3-7}$ Worldwide, we are aware of only one example in day surgery. ${ }^{8}$ Apart from providing participating hospitals with comparative data from other hospitals, a national database can also provide a database for research of specific surgical procedures (which will rarely be performed in sufficiently large numbers in any single hospital), including studies of the factors that are associated with a successful outcome.

This paper describes the development of software to enable hospitals using a standard questionnaire to enter and analyse their own data, the establishment of a national comparative audit database, and some initial results from the first 11 hospitals.

\section{Methods}

ADMINISTRATION OF THE SURVEY

The questionnaire for assessing patients' experiences of day surgery has been described. ${ }^{1}$ It was recommended that the questionnaires be given to 300 consecutive day surgical patients. With an expected response of $65 \%$, information from about 200 patients would be obtained.

As the questionnaires were due for completion about three weeks after surgery they could either be handed to the patients before discharge from hospital or be posted later. A letter from the patient's surgeon or a hospital manager explaining the importance of the survey to the hospital and a stamped addressed envelope accompanied the questionnaire. Given the variety of organisational arrangements in different hospitals, the exact method of carrying out each survey had to be 
decided by the participant. It was recommended that one reminder letter be posted to all non-responders two weeks after the expected date of receipt of the completed questionnaire. By maintaining a register of all patients invited to participate the hospital would be able to assess any non-responder bias, at least in terms of patients' age and sex and procedure.

\section{DEVELOPMENT OF SOFTWARE FOR LOCAI}

USERS

The objective was to produce software that could be used by someone with minimal computing experience. Software for data entry and analysis was prepared with a Paradox relational database system on an IBM compatible personal computer. ${ }^{9}$ A feature of Paradox is that applications can be provided in compiled or run time form so that users do not have to have a copy of the Paradox software itself. A manual was cowritten by the programmer and a member of the team with little computing experience to ensure that it was user friendly. The software was tested in eight sites, and, as a result, some minor modifications were made.

DEVELOPMENT OF COMPARATIVE DATABASE Data received from local users on disk were checked for viruses before being translated into a format which could be read by SPSS/ $\mathrm{PC}+$ version $4 \cdot 0 .{ }^{10}$ Variable and value labels had to be added to the data, frequencies of all variables examined, unusual values recoded, a

\section{Categories of procedure in comparative database}

$(1-20=$ categories included in questionnaire; $21-41=$ additional categories $)$

\section{Ear, nose, and throat \\ 3 Myringotomy or grommets}

6 Nasal fracture reduction

\section{Gynaecological}

13 Sterilisation

18 Termination of pregnancy

19 Dilatation and curretage

20 Cervical cautery/biopsy

39 Colposcopy/hysteroscopy/ transcervical endometrial ablation/intrauterine contraceptive device/cone biopsy

Dental

21 Dental extraction

32 Other oral surgery

Ophthalmic

15 Cataract extraction

\section{Dermatological}

4 Removal of skin growth

22 Toenail removal

29 Skin graft

37 Repair skin lesion

40 Abscess

Investigations

12 Laparoscopy

36 Lumbar puncture/ epidural/myelography

\section{Orthopaedic}

2 Arthroscopy

8 Removal of ganglion

17 Carpal tunnel release

25 Contracture/trigger finger

26 Removal of wire/pin/plate, etc

30 Injection of joint

33 Manipulation of joint

38 Fusion of joint

41 Other orthopaedic procedure

Urological

7 Circumcision

9 Orchidopexy

11 Cystoscopy

14 Vasectomy

23 Prostatic biopsy/urethal dilatation

24 Testicular/penile surgery

Vascular

10 Varicose vein surgery

35 Angiogram

General surgical

1 Hernia repair

5 Anal fissure dilatation/excision

16 Breast lump biopsy

27 Other cysts/lumps/polyps

28 Lower gastrointestinal polyp removal

31 Removal of foreign body

34 Haemorrhoids hospital identifier added to the file, and procedures additional to those listed on the questionnaire had to be coded. The comparative database then had to be backed up and the new data added.

A report for each hospital was produced using SPSS/PC+ tables. One table was produced for each question (or subsection of a question) in the original questionnaire. In each table the responses of patients in the hospital in question were compared with the aggregate responses of patients in the database. The report also included comparisons for specific patient groups. Thus, if the hospital carried out a limited range of procedures on a day basis, then this group of procedures could be selected from the database for comparison.

\section{COMPARATIVE ANALYSES}

All the variables studied were dichotomous. The proportion of patients expressing an unfavourable outcome or dissatisfaction in each hospital, together with $95 \%$ confidence intervals, were compared.

\section{Results}

SOFTWARE FOR LOCAL USERS

Software was written which enabled users of the questionnaire to enter their data on their own personal computers. Validity checks were programmed into the software to ensure that logically inconsistent responses could not be entered. Users were also provided with a series of standard analyses, which included frequency distributions of all the variables for which information was collected, plus cross tabulations that compared the overall level of satisfaction with sociodemographic factors; preoperative anxiety; and outcome (pain, wound problems, change in symptoms, and daily activities). The standard analyses could be stored on the disk or printed.

In addition, local users could specify and carry out their own analyses with the software. Alternatively, a data file could be created and exported in various different formats for analysis with other more advanced software packages. Back up data files could be created on floppy disks and this facility could be used to send data away for inclusion in the comparative database.

\section{COMPARATIVE DATABASE}

Data from the first 11 hospitals to use the questionnaire were obtained. The mean number of patients responding was 158 (range 64 to 269). The overall response rate was $60 \%$ but varied from $33 \%$ to $90 \%$. Hospitals with low response rates had made no attempt to remind non-responders whereas those with the highest rates had pursued non-responders by repeated telephone calls. Most hospitals had investigated a broad mix of patients, though one had chosen to survey only patients undergoing cataract surgery.

The data received on floppy disks from each hospital were prepared, added to the main database, and a report specific for the hospital was printed and dispatched within a few days. 


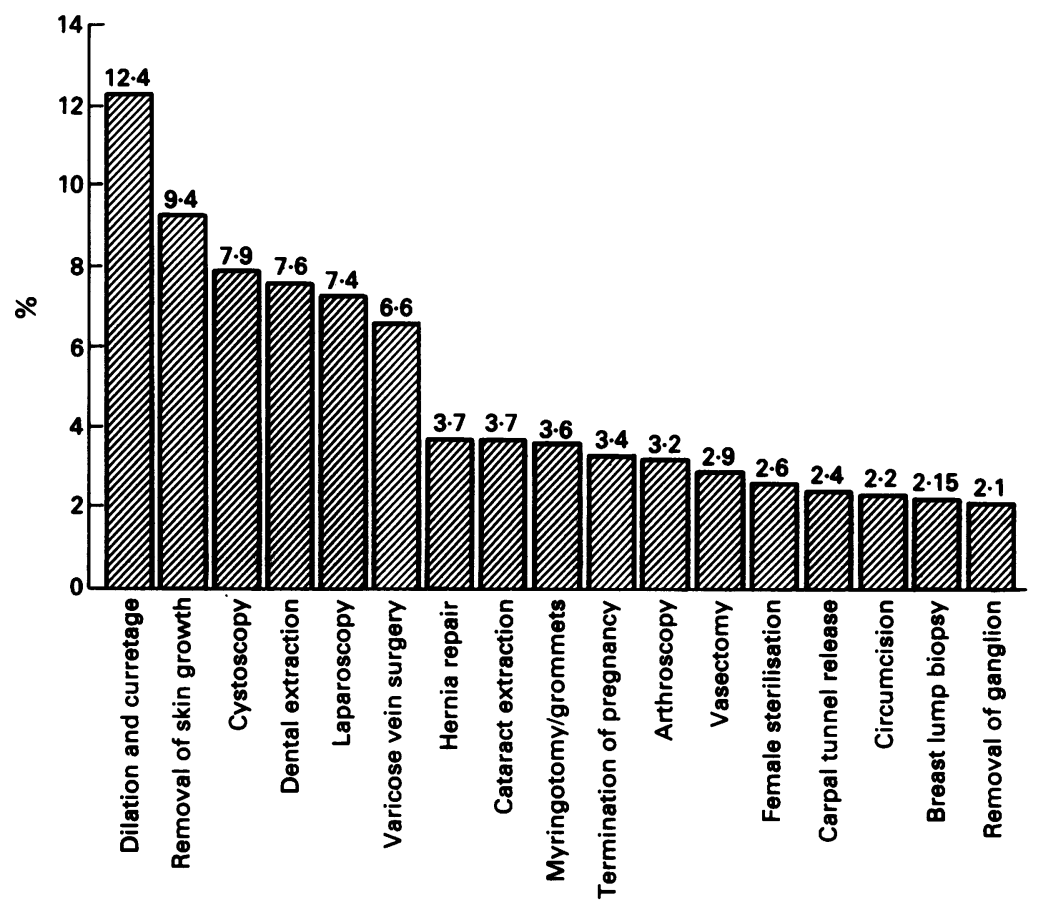

Fig 1 Procedures accounting for at least $2 \%$ of all day surgery procedures $(n=1741)$

Though SPSS/PC+ was slow to perform the calculations required to generate the tables (and around 600 lines of SPSS program code were required to generate the report), it had the advantage that the "tables" procedure was flexible enough to allow considerable control over the layout and contents of the output. This meant that the resulting tables could be printed directly without further editing with a word processing package.

Each report contained 72 tables and a series of bar charts based on selected tables from the report. These charts displayed comparisons between the individual hospital and the national database and covered a range of aspects of day surgery, including satisfaction with the process of care, frequency of complications, effectiveness of the operation, and overall satisfaction.

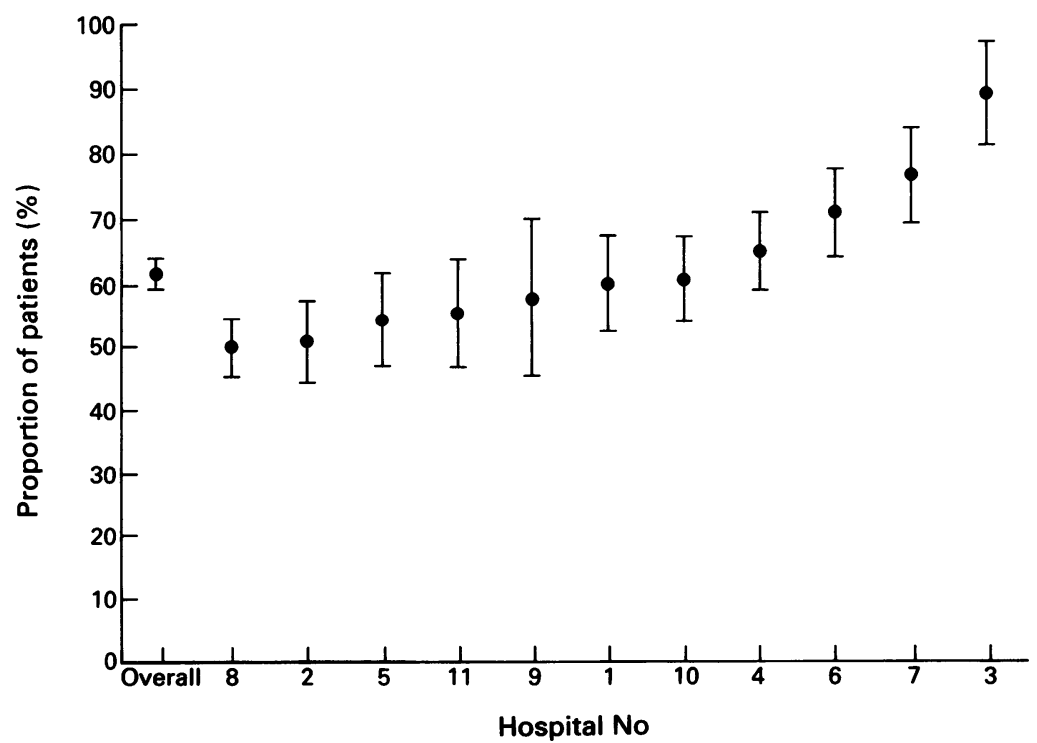

Fig 2 Proportion of patients receiving written information before admission, by hospital; bars are $95 \%$ confidence intervals
INITIAL COMPARISONS AMONG HOSPITALS Many patients $(28 \%)$ reported having undergone procedures other than those listed on the questionnaire. Most of these procedures were coded by the authors to either one of the existing 20 categories or one of 21 additional categories (box), leaving only 3\% uncoded. Patients who had undergone more than one procedure $(6 \%)$ were categorised according to the major procedure. For example, dilatation and curretage was considered to be more major than cervical cautery.

Figure 1 shows those procedures that accounted for at least $2 \%$ of the total number of procedures. Seventeen procedures accounted for $83 \%$ of all those performed. Some procedures would not usually be considered as day case procedures but could have been carried out appropriately in an outpatient setting. These included most dental extractions $(7 \cdot 6 \%$ of total); toenail removal $(1 \cdot 8 \%)$; and removal of various cysts, lumps, and stitches $(1 \cdot 4 \%)$. In all, up to $12 \cdot 3 \%$ of day case patients might have had their procedure performed in outpatient departments. This varied between hospitals from $0 \%$ to $27 \%$.

Figures 2-5 show some of the differences observed between hospitals. The proportion of patients who received written information before admission ranged from $50 \%$ to $89 \%$ (fig 2 ); overall, about a third of all patients did not receive written information. Most hospitals provided adequate parking facilities, but there were two obvious exceptions (fig 3). In contrast, there was little difference between hospitals in patients' overall level of satisfaction (fig 4). Finally, the proportion of patients experiencing a significant amount of pain after surgery ranged from $8 \%$ to $42 \%$ (fig 5 ). None of these variations took differences in case mix (age, sex, or procedure) into account.

\section{Discussion}

The work reported here has shown that it is feasible to establish a comparative database and produce reports of day surgery for specific hospitals. However, it has also illustrated several methodological problems that need to be overcome if the full potential of such an approach is to be realised.

The first is the need to improve the response rates to the questionnaire in some hospitals. With dedication rates of $90 \%$ can be achieved. Though the tools for carrying out a survey can be provided from a central agency, clinicians and managers locally must appreciate the need to commit realistic resources to the exercise. Without these resources the survey will at best be a waste of time and at worst produce misleading results which might lead to erroneous management decisions. Identifying all consecutive day surgery patients, providing them with questionnaires, maintaining a register of responses, pursuing non-reponders, entering the responses, and producing the standard suite of analyses will entail more time than a member of the clerical staff is likely to have in addition to his or her existing work. If the information gained from a survey is valued by the clinicians and managers then a member 


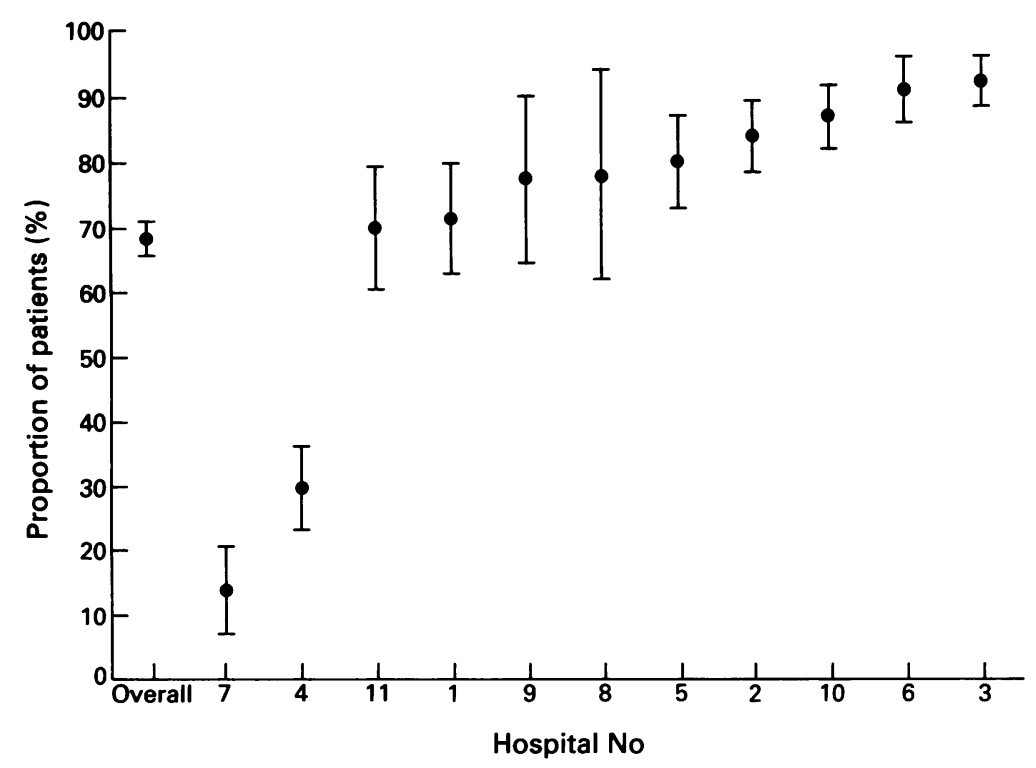

Fig 3 Proportion of patients satisfied with parking facilities, by hospital; (excluding those answering "not applicable") bars are $95 \%$ confidence intervals

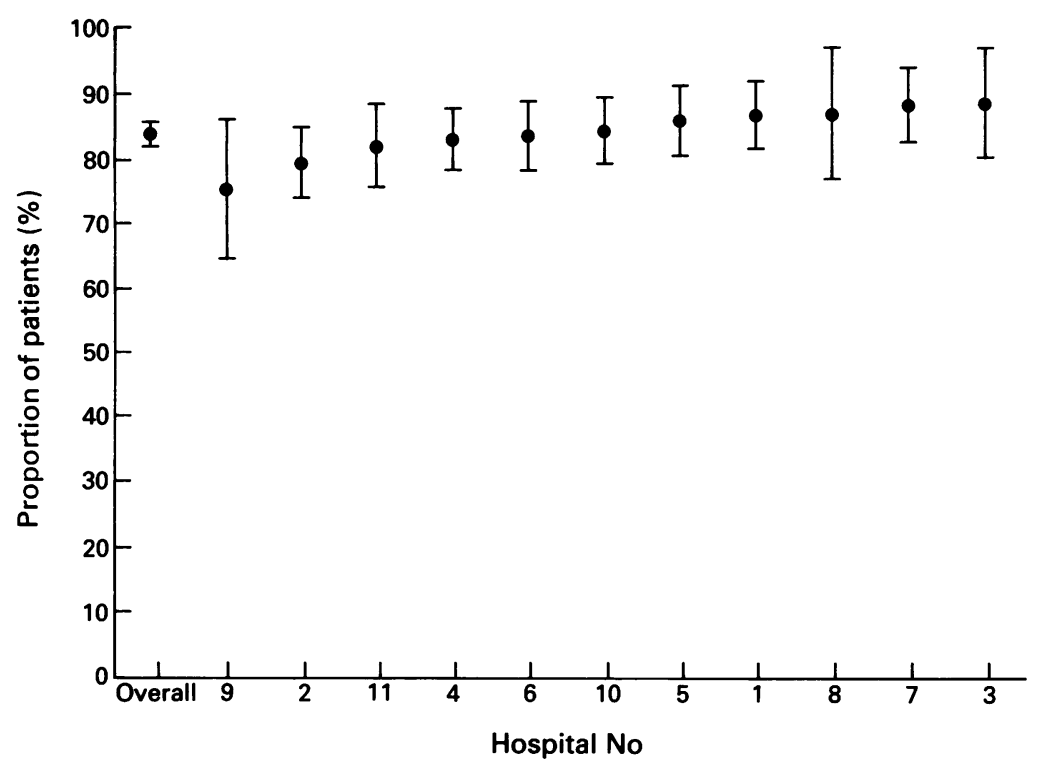

Fig 4 Proportion of patients who would recommend day surgery in similar circumstances, by hospital; bars are $95 \%$ confidence intervals

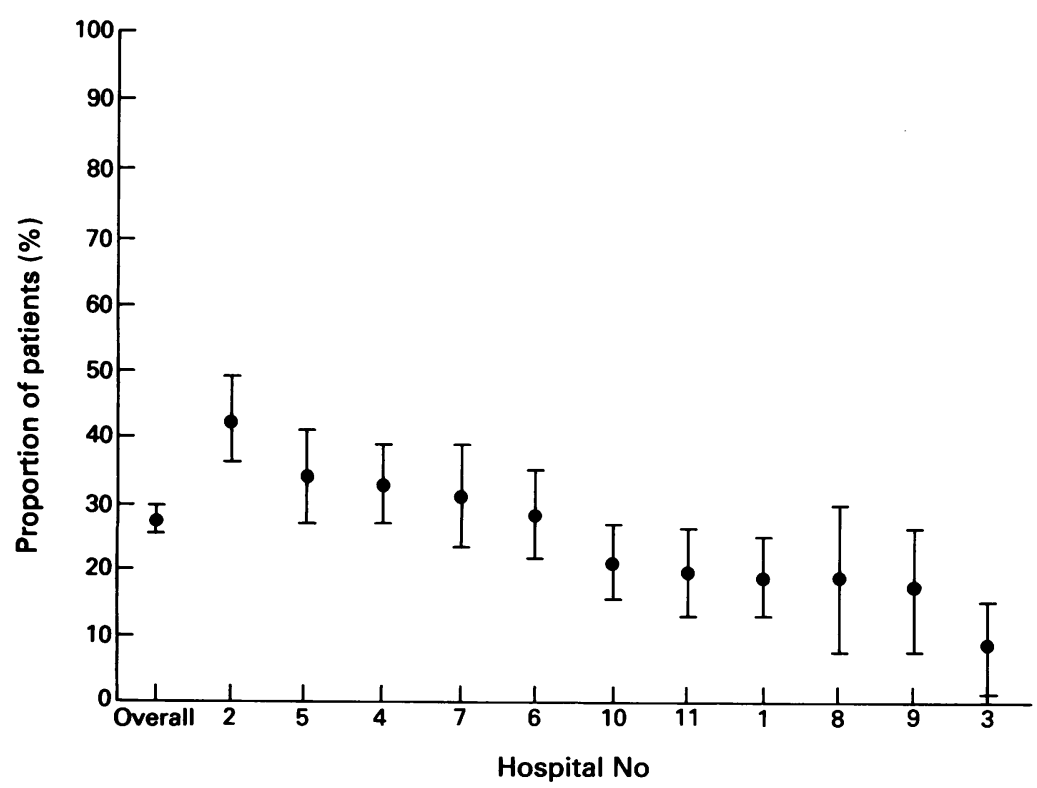

Fig 5 Proportion of patients experiencing a significant amount of pain in the first 24 hours postoperatively, by hospital; bars are $95 \%$ confidence intervals of staff must be allocated the task and given sufficient time to do it properly. This clearly happened in some of the 11 participant hospitals but not in others.

The second problem is the need to account for differences in case mix. Though case mix should not have much impact on measures such as satisfaction with parking facilities (fig 3) or receiving written information (fig 2) it may, for example, affect the proportion of patients experiencing postoperative pain (fig 5). In view of the large number of outcome measures collected covering both the effectiveness and humanity of care, it may be necessary to develop several methods for adjusting case mix, each one specific to a group of outcome measures. Work is in progress to develop a valid method of case mix adjustment, which seems never to have been done for patient reported outcome and satisfaction.

The third problem is that of sample size. Difficulties of collecting sufficiently large samples for auditing the care of specific patient groups have been found with even a common surgical procedure such as appendicectomy. ${ }^{11}$ As the comparative database expands it will be possible to carry out analyses of specific procedures - something that is unlikely for any individual hospital in view of the few patients undergoing any one procedure in a short period. It is planned to produce a series of technical publications for the participating hospitals on each of the commonest procedures. In time it will even be possible to report on the less frequently performed procedures.

Finally, the principal purpose of establishing a comparative audit facility is to help to improve the quality of day surgery. This will occur only if the reports provided for participating hospitals are thought to be useful by the staff and managers and are seen to have some impact in bringing about change. We plan to assess these aspects in a larger sample of hospitals. By early 1993 over 50 hospitals had requested the questionnaire and local software; data from over 20 of them had been included in the comparative database.

If these methodological challenges can be met comparative audit offers opportunities for evaluative research in addition to those for audit. Firstly, the database can be used to generate hypotheses which can then be tested with experimental methods. Secondly it can be used to detect rare effects of interventions that will only be apparent when large numbers of cases are observed. Thirdly, it can be used to compare packages of care that are difficult to subject to experimental research designs. Finally, it offers the opportunity to study a broad cross section of medical practice rather than confining research to a small, possibly unrepresentative, group of practitioners.

We thank Dr Jonathan Boyce, John Bailey, and Linda Jarrett of the Audit Commission for their help and support; Dr Lucy Moore for help in establishing the comparative database; and Moore for help in establishing the comparative database; and
the Audit Commission for funding this work. The questionthe Audit Commission for funding this work. The question-
naires and local software are available from the Audit naires and local software are available from the Audit Gifford, Bristol BS12 62U. Inquiries about the comparative database to Dr Mark Petticrew, Health Services Research Unit, London School of Hygiene and Tropical Medicine, London WC1E 7HT. 
1 Black NA, Sanderson CFB. Day surgery: development of in Health Care 1993;2:157-61.

2 Black NA. A regional computerised surgical audit project. Quality Assurance in Health Care 1990;2:263-70.

3 English TAH, Bailey AR, Dark JF, Williams WG. The UK cardiac surgical register, 1977-82. BMF 1984; 289.1205-08.

4 Taylor RMR, Ting A, Briggs JD. Renal transplantation in the United Kingdom and Ireland - the centre effect. the United Kingdom and Ireland - the centre effect.

5 Gruer R, Gordon DS, Gunn AA, Ruckley CV. Audit of surgical audit. Lancet $1986 ; \mathrm{i}: 23-6$.

6 Dunn DC, Dale RF, Gumpert JRW, Duffy TJ. Combined surgical audit by microcomputer involving units in four health regions. Ann $R$ Coll Surg Engl 1992;74:47-53.

7 Dunn DC, Fowler S. Comparative audit: an experimenta study of 147882 general surgical admissions during 1990. Br f Surg 1992;79:314-6.

8 Steven ID. A patient satisfaction questionnaire as a teaching and comparative audit tool. Quality Assurance in Health Care 1991;3:41-9.

9 Borland International. Paradox Version 3.5. United States: Borland International, 1990.

10 SPSS International SPSS/PC + Version 4.0. Netherlands: SPSS International, 1990.

11 Black NA, Moore L. Comparative audit between hospitals: the example of appendicectomy. International fournal of Quality Assurance in Health Care. (in press).
列 